\section{ORIGINAL RESEARCH}

B. Meissner

K. Kallenberg

P. Sanchez-Juan

A. Krasnianski

U. Heinemann

D. Varges

M. Knauth

I. Zerr

\title{
Isolated Cortical Signal Increase on MR Imaging as a Frequent Lesion Pattern in Sporadic Creutzfeldt-Jakob Disease
}

BACKGROUND AND PURPOSE: Hyperintense basal ganglia on MR imaging support the diagnosis of sporadic Creutzfeldt-Jakob disease (CJD). Our aim was to study the frequency of patients with sporadic CJD presenting with and without characteristic basal ganglia lesions on MR imaging and to examine the corresponding patient characteristics.

MATERIALS AND METHODS: Fluid-attenuated inversion recovery (FLAIR) and diffusion-weighted images (DWI) of 55 patients with CJD were assessed for signal-intensity increase (FLAIR) or restricted diffusion (DWI) in 7 cortex regions and the basal ganglia, thalamus, and cerebellum. Patient characteristics as well as electroencephalography, CSF, and codon 129 genotype of the prion protein gene (PRNP) were correlated with the most frequent MR imaging lesion patterns.

RESULTS: Two major lesion patterns were identified by DWI: cortex and basal ganglia involvement (two thirds) and isolated cortex involvement (one third). In the latter patient group, the cortex involvement was widespread (at least 3 regions affected in $89 \%$ on DWI) and usually included the frontal and parietal lobes (78\%). The length of the disease course was significantly prolonged (median, 12 versus 5 months). No significant differences were observed concerning electroencephalography and CSF findings and codon 129 genotype distributions. Of 4 patients with normal MR imaging findings, the CSF was positive for the 14-3-3 protein in 3.

CONCLUSION: A high number of patients with CJD present without basal ganglia lesions on MR imaging. Isolated cortex involvement on DWI and FLAIR should lead to suggestion of CJD, even if the disease course is only slowly progressive. Additional 14-3-3 protein analysis in the CSF may support the CJD diagnosis.

$\mathbf{S}^{p}$ poradic Creutzfeldt-Jakob disease (CJD) is a rare and fatal disease caused by the accumulation of abnormal/pathologic prion protein ( $\mathrm{PrP}^{\mathrm{Sc}}$; Sc indicates scrapie) in the human brain. The classic disease type is characterized by rapidly progressive dementia, ataxia, abnormal muscle tone, and myoclonus. It leads to a state of akinetic mutism and death after a median disease duration of 6 months. ${ }^{1}$ The definite CJD diagnosis relies on the finding of $\mathrm{PrP}^{\mathrm{Sc}}$ in the brain tissue, together with astrocytic gliosis, nerve cell loss, and spongiform degeneration as the typical neuropathologic changes. ${ }^{2,3}$

During one's lifetime, MR imaging hyperintensity of the basal ganglia on T2-weighted (T2WI), fluid-attenuated inversion recovery (FLAIR), and diffusion-weighted imaging (DWI) is increasingly used to support the CJD diagnosis, next to positive CSF (14-3-3 protein) and electroencephalography (EEG) findings of periodic sharp-wave complexes (PSWCs).

\section{Received December 25, 2007; accepted after revision March 12, 2008.}

From the National TSE Reference Center at the Department of Neurology (B.M., A.K., U.H., D.V., I.Z.) and Department of Neuroradiology and MR Research in Neurology and Psychiatry (K.K., M.K.), Georg-August University of Göttingen, Göttingen, Germany; Foundation Marqués de Valdecilla (P.S.-J.), El Instituto de Formación e Investigación Marqués de Valdecilla (IFIMAV), Santander, Spain; Centro de Investigación Biomédica en Red sobre Enfermedades Neurodegenerativas (CIBERNED) (P.S.-J.); and Department of Gerontopsychiatry (B.M.), University of Zürich, Zürich, Switzerland.

Authors B.M. and K.K. contributed equally to this work.

This work was supported by grants from the Federal Ministry of Education and Research (BMBF 01GI0301 and KZ: 0312720), Federal Ministry of Health (BMG Az325-4471-02/15), and Robert Koch Institute through funds of the Federal Ministry of Health (grant no. 1369-341).

Please address correspondence to Bettina Meissner, MD, Department of Gerontopsychiatry, University of Zürich, Minervastr. 145, CH-8008 Zürich, Switzerland; e-mail: bettina.meissner@puk.zh.ch

DOI 10.3174/ajnr.A1122
Although the origin of the signal-intensity changes is still not fully understood, hyperintensity on T2WI and FLAIR has been thought to be caused by gliosis, whereas abnormalities on DWI are most likely derived from spongiform changes. ${ }^{4-6}$ DWI was shown to be the most sensitive sequence in the detection of brain lesions, particularly in the neocortex. ${ }^{7-10}$ Isolated cortex involvement was also found. ${ }^{9,11}$

Although abnormal MR imaging findings in CJD have been studied in detail with respect to their location, few attempts have been made to define the most frequently occurring patterns of hyperintensity in a spectrum of patients. Six disease phenotypes (MM1, MM2, MV1, MV2, VV1, and VV2) defined by the codon 129 genotype (MM, MV, VV) of the prion protein gene $(P R N P)$ and pathologic isotype of the $\mathrm{PrP}^{\mathrm{Sc}}$ type 1 or 2 have been recently described with distinctive neuropathologic features and various clinical and diagnostic findings. ${ }^{1-3,12}$ On MR imaging, predominant cortical (VV1) ${ }^{13}$ or subcortical involvement (MV2 and VV2 $)^{14,15}$ or no abnormalities (MM2) ${ }^{16,17}$ were found in smaller case series.

To date, to our knowledge, the overall distribution of MR imaging abnormalities has not been studied in a larger spectrum of patients with CJD, and it is unclear whether there are clinical correlates corresponding to specific MR imaging lesion patterns. The proportion of patients presenting without basal ganglia abnormalities is unknown.

We defined the most frequent MR imaging lesion patterns and corresponding clinical characteristics in a CJD patient collective by using highly sensitive MR images, and we considered a possible influence of the codon 129 genotype of the PRNP. We particularly focused on patients lack- 
ing basal ganglia abnormalities on MR imaging and suggested criteria that might support the early CJD diagnosis in these patients.

\section{Materials and Methods}

\section{Patients}

Patients with suggested CJD in Germany are reported to the National TSE (Transmissible Spongiform Encephalopathy) Reference Center in Göttingen. After notification, these patients are examined by a study physician at the hospital reporting the patient. Copies are made of the medical charts (clinical findings, laboratory tests) as well as EEG and MR imaging findings, and a neurologic examination is performed. According to the current WHO criteria, ${ }^{18}$ the diagnosis of possible CJD requires progressive dementia and 2 of 4 typical clinical symptoms (visual and/or cerebellar and pyramidal and/or extrapyramidal symptoms, myoclonus, and akinetic mutism). For probable CJD, characteristic EEG changes (PSWCs) or the presence of the 143-3 protein in the CSF or both are additionally required. The definite CJD diagnosis relies on postmortem examination.

The study collective was searched from the years 2000 to June 2005 (predominant use of DWIs from the year 2000 on) by using the MR imaging data base of the CJD Surveillance Unit, Göttingen, Germany. Patients with definite or probable CJD diagnosis for whom a FLAIR and DWI were available from the same examination date were included in the study. Genetic and iatrogenic (CJD) cases were excluded.

\section{Neuropathology}

Histologic examination was performed on $4-\mu$ m-thick sections of formalin-fixed and paraffin-embedded brain tissue blocks. Hematoxylin and eosin stains, as well as immunochemistry, were performed by using standard techniques. Monoclonal antibodies Gö 138 and L42 were used for immunohistochemical detection of $\operatorname{PrP}^{\mathrm{Sc}}$.

\section{Genetic Analysis}

Analysis of the PRNP was performed after isolation of genomic deoxyribonucleic acid from blood according to standard methods. ${ }^{19}$ The codon 129 genotype of the PRNP (MM, MV, and VV) was available in 48 of 55 patients.

\section{EEG and CSF}

EEG recordings were available in 48 patients and were assessed as positive or negative for PSWCs according to the established criteria (generalized periodic sharp waves with $0.5-2 / \mathrm{s}$ frequency, 100- to 600-ms duration, and $>150-300 \mu \mathrm{V}$ of amplitude). ${ }^{20} \mathrm{CSF}$ samples were available in all 55 patients. The 14-3-3 protein immunoassays by means of Western blotting were performed at least twice in each sample as described previously. ${ }^{21}$

\section{MR Imaging}

The MR images were collected from various hospitals in Germany (in the framework of the epidemiologic study) and obtained from scanners of different manufacturers (mainly Siemens, Erlangen, Germany; Philips Medical Systems, Best, the Netherlands; Marconi Medical Systems, Cleveland, Ohio; Picker International, Cleveland, Ohio; and GE Medical Systems, Milwaukee, Wis) and of different magnetic field strengths of $0.5-2.9 \mathrm{~T}$ (mainly $1.5 \mathrm{~T}$ ). The manufacturer's standard sequences were applied. The individual technique (single-shot, multishot, echo-planar imaging, stimulated echo) and the b-values (up to
4 b-values: $0.333,666,1000$, maximal $1500 \mathrm{~s} / \mathrm{mm}^{2} ; b=1000 \mathrm{~s} / \mathrm{mm}^{2}$ was most common) depended on the local facility (eg, gradient system) and individual preferences. All scans were assessed by a neuroradiologist (K.K.) who was aware of the CJD diagnosis. Because the reliability of the neuroradiologic evaluation was reported to be high, the scans were rated by 1 neuroradiologist only. ${ }^{8}$ For each patient, the FLAIR-weighted scan was assessed first, followed by DWI. Protonattenuation-weighted scans and apparent diffusion coefficient maps were less commonly used and were not included in this study. The brain regions were evaluated as hyperintense in relation to suspected normointense brain tissue according to a standardized protocol, including 7 cerebral cortex regions (frontal, parietal, temporal, occipital regions and the cingulate gyrus, insula, and hippocampus), basal ganglia (caudate nucleus, putamen, globus pallidus), thalamus, and cerebellar cortex. The quality of the complete MR imaging examination was graded from 1 to $6(1=$ excellent, $2=$ good, $3=$ average, $4=$ sufficient, 5 = insufficient, 6 = poor), according to individual subjective criteria (eg, due to motion or metal), technique (sequence parameters: T2WI half-Fourier acquired single-shot turbo spin-echo protocol of the brain, acquisition time, resolution, inversion recovery pulse), homogeneity, differentiability of gray and white matter, postprocessing (eg, filtering, smoothing), and, in case of hard copies, also the selected window and the absolute image size. Scans graded worse than 4 were considered as nondiagnostic and excluded from the study. If serial MR images were available, the first examination was used for the study.

\section{Statistical Analysis}

For statistical analysis, the program Statistica was used. Fisher exact and Mann-Whitney $U$ tests were used to assess differences between qualitative and quantitative variables, respectively. For comparison of brain regions showing abnormal signal intensity on FLAIR or restricted diffusivity on DWI, the McNemar test was used.

\section{Results}

\section{Study Collective}

In the years 2000 to June 2005, 55 patients with probable ( $n=$ $24)$ or definite $(n=31)$ sporadic CJD fulfilled the inclusion criteria. The group consisted of 28 men and 27 women. The median age of all patients was 63 years (range, 29-79 years), and the median disease duration was 6 months (range, 1-41 months). Analysis of the codon 129 genotype most frequently revealed the MM genotype (63\%), followed by MV (25\%), and VV (13\%) (Table 1).

\section{MR Imaging}

MR imaging was performed after a median of 3 months (range, 10 days to 22 months) after the onset of symptoms. The overall quality of the scans was graded as good (overall score $=2$ ). A synopsis of the MR imaging findings as detected by FLAIR and DWI is given in Table 2. DWI was the most sensitive technique, showing basal ganglia lesions in $60 \%$ ( $47 \%$ on FLAIR) and cortical lesions in $91 \%$ ( $76 \%$ on FLAIR). Lesions were spotted as early as 10 days after the onset of symptoms (cortex and basal ganglia on DWI and FLAIR). In 5 of 55 patients, lesions were only visible on DWI (3 isolated cortical lesions, 2 cortex and basal ganglia lesions). In 4 patients, basal ganglia lesions were seen only on DWI. In 2 patients, only FLAIR showed this finding. 


\begin{tabular}{|c|c|c|c|c|}
\hline Patient Characteristics & All Patients $(n=55)$ & Cortex and Basal Ganglia $(n=32)$ & Only Cortex $(n=18)$ & $P$ Value \\
\hline Male/female & $28 / 27$ & $17 / 15$ & $9 / 9$ & .83 \\
\hline Median age (yr) & 63 & 63 & 62 & .20 \\
\hline Range (yr) & $29-79$ & $41-79$ & $46-74$ & \\
\hline Median duration (mo) & 6 & 5 & 12 & .05 \\
\hline Range (mo) & $1-41$ & $1-41$ & $3-32$ & \\
\hline \multirow[t]{3}{*}{ Codon 129 genotype of the PRNP } & $\mathrm{MM}(64 \%)$ & $\mathrm{MM}(62 \%)$ & $\mathrm{MM}(67 \%)$ & .09 \\
\hline & MV $(25 \%)$ & MV $(24 \%)$ & MV (27\%) & \\
\hline & W (12\%) & VV (14\%) & VV (7\%) & \\
\hline MR m.a.o. & 3.0 & 2.2 & 3.6 & .23 \\
\hline Range & $0.3-22.0$ & $0.3-19.8$ & $1.0-22.0$ & \\
\hline MR m.b.d. & 3.0 & 1.8 & 8.0 & .06 \\
\hline Range & $0.3-23.1$ & $0.3-23.1$ & $0.4-22.8$ & \\
\hline CSF & $85 \%$ & $81 \%$ & $94 \%$ & .40 \\
\hline $14-3-3+$ & $46 / 54$ & $26 / 32$ & $17 / 18$ & \\
\hline EEG & $31 \%$ & $26 \%$ & $36 \%$ & .49 \\
\hline PSWC+ & $15 / 49$ & $8 / 31$ & $5 / 14$ & \\
\hline
\end{tabular}

Note:-m.a.o. indicates months after onset; m.b.d., months before death; DWI, diffusion-weighted imaging; EEG, electroencephalography; PSWC, periodic short-wave complex. * In 1 patient, only basal ganglia signal increase was found; 4 patients did not show signal alterations on the MR Imaging.

\begin{tabular}{lcrr}
\hline \multicolumn{4}{l}{ Table 2: MR imaging sensitivity: comparison of FLAIR and DWI } \\
\hline Hyperintense Brain Regions & FLAIR & \multicolumn{1}{c}{ DWI } & FLAIR or DWI \\
\hline Basal ganglia & $26(47 \%)$ & $33(60 \%)$ & $35(64 \%)$ \\
Cortex & $42(76 \%)$ & $50(91 \%)$ & $50(91 \%)$ \\
Thalamus & $4(7 \%)$ & $6(11 \%)$ & $6(11 \%)$ \\
Cerebellum & $14(25 \%)$ & $17(30 \%)$ & $22(40 \%)$
\end{tabular}

Note:-FLAIR indicates fluid-attenuated inversion recovery; DWI, diffusion-weighted imaging.

In 8 of 55 patients, cortical involvement was displayed only on DWI. In these patients, the frontal lobes were affected most frequently (7/8), followed by the cingulate gyrus (5/8) and the temporal and parietal lobes (4/8, each). In comparison with FLAIR, DWI was significantly more sensitive for the detection of cortical lesions in general $(P=.01333)$ and with respect to the frontal, temporal, parietal, insular, and occipital cortices (Fig 1).

\section{MR Imaging Lesion Patterns and MR Images}

The most frequent MR imaging lesion patterns were defined by using DWI as the most sensitive technique (Fig 2). Cortex and basal ganglia hyperintensity was observed in two thirds (58\%) and isolated cortical hyperintensity, in one third (33\%) (Fig 3). Normal MR imaging findings were seen in 4 patients (7\%).

The MR imaging lesion patterns obtained for each sequence are summarized in Table 3.

Findings on FLAIR and DWI were not completely congruent: The FLAIR sensitivity for the cortex and basal ganglia was reduced (compared with patients showing that pattern on DWI), because in 3 patients, only basal ganglia hyperintensity was found, and in 2 patients, no signal-intensity changes were detected. Six patients with isolated cortex involvement on FLAIR showed basal ganglia lesions on DWI. Three patients with isolated cortical hyperintensity on DWI had normal findings on FLAIR images.

\section{Isolated Cortical Hyperintensity on the MR Imaging}

The most frequently observed patterns of cortical hyperintensity are given in Table 4 . On DWI, the frontal and parietal lobes were most frequently affected ( $89 \%$, each), followed by the temporal (72\%) and occipital lobes (61\%) (data not shown). The cortical lesions were widespread, including at least 3 regions in $89 \%$ of the patients. The frontal and parietal lobes were most frequently affected in combination (78\%) (Fig 3). Hyperintensity of only 1 region (the temporal lobe) on DWI was found in a single patient.

On FLAIR, the frontal lobes (79\%) were most frequently affected, followed by the parietal lobes and the cingulate gyrus (63\% each) (data not shown). Combined hyperintensity in the frontal and temporal lobes was the most frequently occurring pattern (53\%). A higher rate of signalintensity increase in the hippocampus $(42 \%)$ was found compared with DWI (28\%).

\section{MR Imaging Lesion Patterns and Clinical Findings}

Clinical data on patients with various MR imaging lesion patterns on DWI are displayed in Table 1. In patients with cortex and basal ganglia hyperintensity on MR imaging, the disease course was rapid, whereas in patients with isolated cortical hyperintensity, the disease duration was significantly prolonged (median, 12 versus 5 months, $P=.05$ ). No significant differences were found concerning the age at disease onset, sex, codon 129 genotype, EEG, CSF, and time point of the MR imaging examination $(P=.23)$. In 4 of 55 patients, no abnormalities apart from atrophy were found on the MR imaging. The disease duration in these patients was variable (3-38 months). One patient was very young at disease onset (female, 29 years, MM genotype) and had the longest disease duration. The CSF was positive for the 14-3-3 protein in 3 of these patients; EEG was positive for PSWC in 1.

\section{Degree of Cortex Involvement and Disease Duration}

A possible correlation between the degree of cortex involvement and the disease duration was examined for FLAIR and DWI (data not shown). Patients with $>3$ cortex regions affected on FLAIR had a significantly prolonged disease duration compared with patients with $<3$ regions affected (median, 13 versus 4 months, $P=.02$ ). On DWI, no such correlation was found (median, 8 versus 6 months, $P=$ $.68)$. 


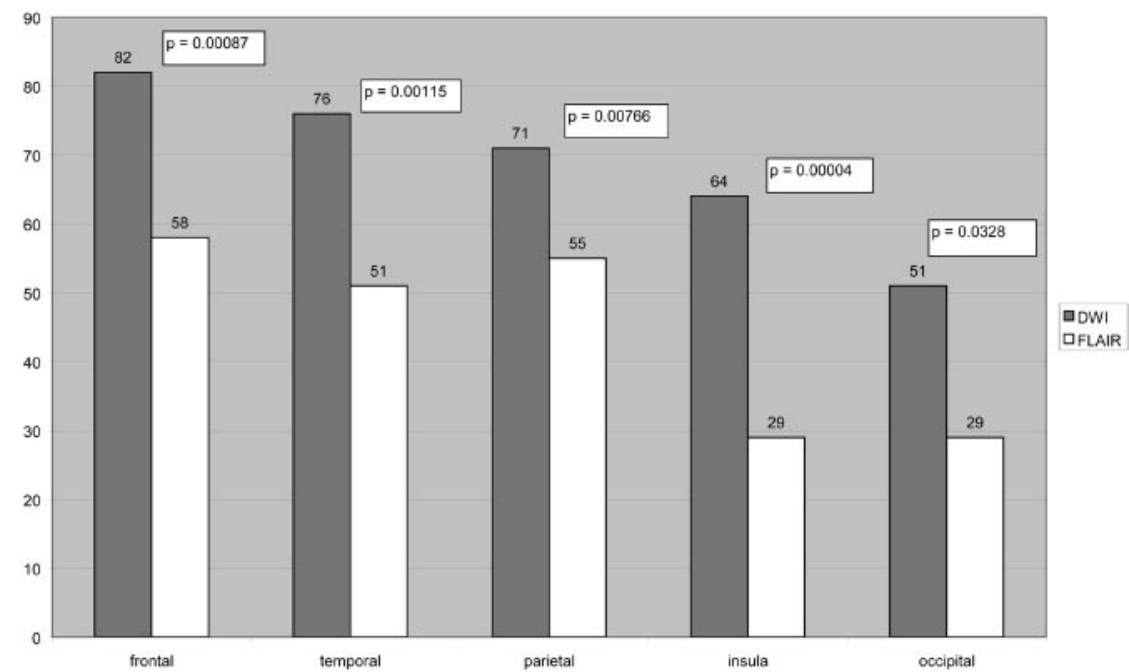

Fig 1. Detection of cortical hyperintensities by FLAIR and DWI in 55 patients with CJD. (The cingulate gyrus and hippocampus are not displayed because the findings were not significant.)
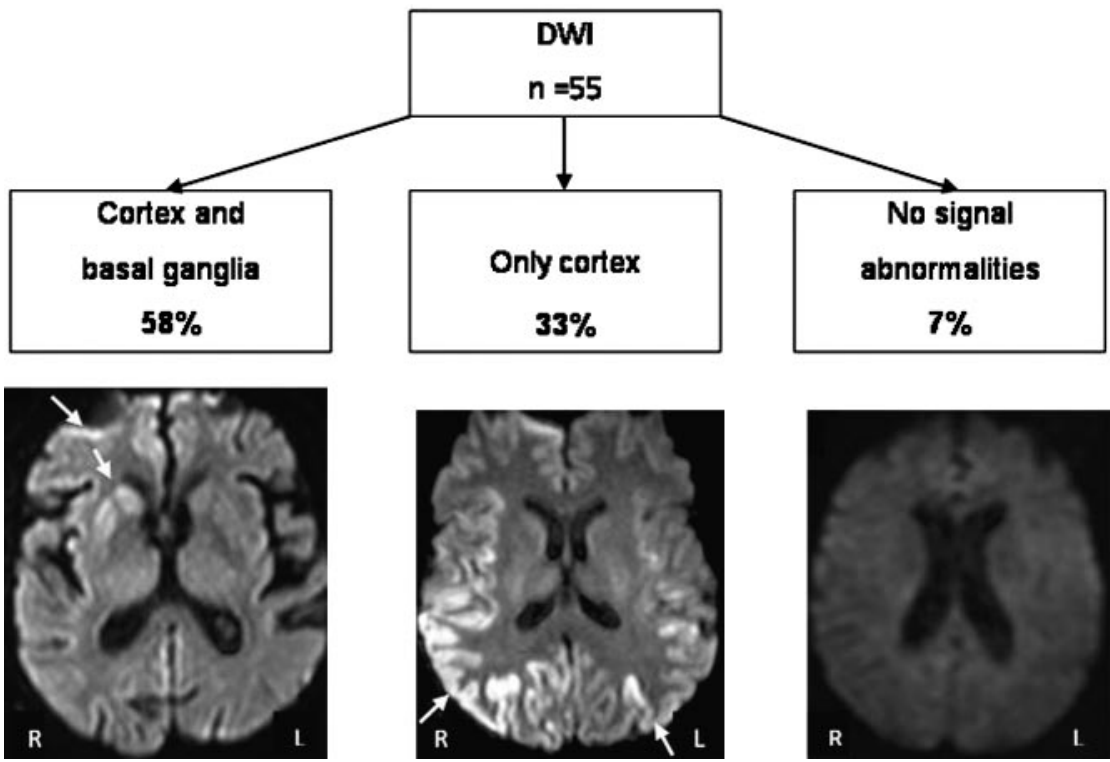

Fig 2. MR imaging lesion patterns in sporadic CJD depicted by DWI.
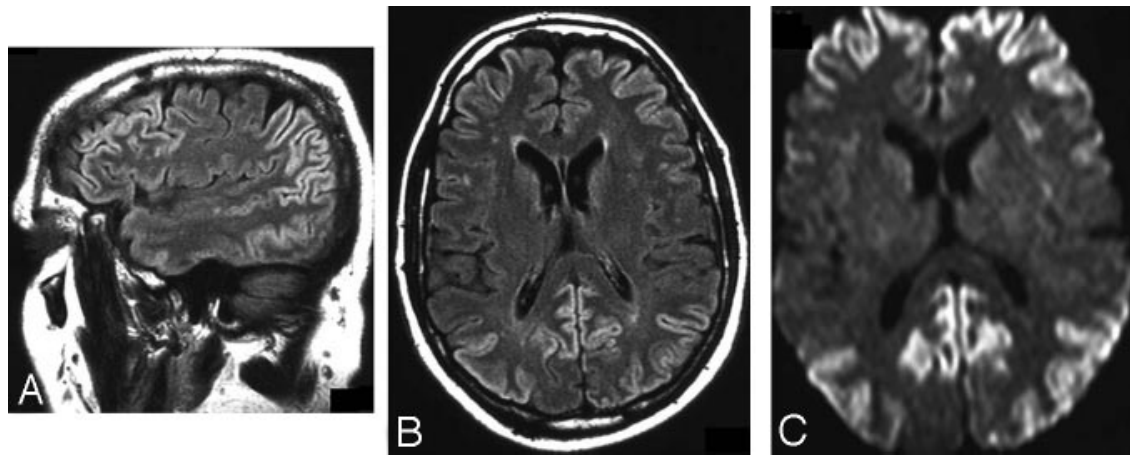

Fig 3. Involvement of the frontal and parietal lobes in patients with isolated cortical hyperintensities. A, Sagittal FLAIR-weighted scan of a 58 -year-old patient 3.5 months after the disease onset and 1 week before death showing signal-intensity increases of the frontal and parietal lobes. B, Axial FLAIR-weighted scan of the same patient showing frontal and parietal

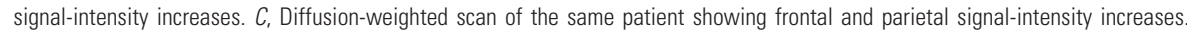

\section{Discussion}

We analyzed the spectrum of MR imaging and clinical findings in a collective of CJD patients with FLAIR and DWI available and were able to define 2 major lesion patterns: combined cortex and basal ganglia hyperintensity (two thirds of all patients) and isolated cortical involvement (one third). In the latter patients, the disease course was significantly prolonged compared with patients with cortex and basal ganglia lesions. 


\begin{tabular}{|c|c|c|c|c|}
\hline \multicolumn{5}{|c|}{$\begin{array}{l}\text { Table 3: Spectrum of MR imaging lesion patterns in } 55 \text { patients } \\
\text { with sporadic CJD }\end{array}$} \\
\hline & \multicolumn{2}{|c|}{$\begin{array}{l}\text { Basal Ganglia } \\
\text { Involvement }\end{array}$} & \multicolumn{2}{|c|}{$\begin{array}{l}\text { No Basal Ganglia } \\
\text { Involvement }\end{array}$} \\
\hline Sequence & $\begin{array}{l}\text { Cortex and Basal } \\
\text { Ganglia }\end{array}$ & $\begin{array}{l}\text { Only Basal } \\
\text { Ganglia }\end{array}$ & Only Cortex & Normal $^{*}$ \\
\hline$\overline{\mathrm{DWI}}$ & $32(58 \%)$ & $1(2 \%)$ & $18(33 \%)$ & $4(7 \%)$ \\
\hline FLAIR & 23 (42\%) & $3(5 \%)$ & $19(35 \%)$ & $10(18 \%$ \\
\hline Total & \multicolumn{2}{|c|}{ DWI $(60 \%)$} & \multicolumn{2}{|c|}{ DWI $(40 \%)$} \\
\hline & \multicolumn{2}{|c|}{ FLAIR $(47 \%)$} & \multicolumn{2}{|c|}{ FLAIR $(53 \%)$} \\
\hline
\end{tabular}

Note:-DWI indicates diffusion-weighted imaging; FLAIR, fluid-attenuated inversion recovery.

${ }^{*}$ Atrophy was found in most patients

Table 4: Distribution of cortical lesions in patients with isolated cortical hyperintensity

\begin{tabular}{lcc}
\hline & DWI $(n=18)$ & FLAIR $(n=19)$ \\
Isolated Cortex Involvement & $(\%)$ & $(\%)$ \\
\hline$\geq 2$ Regions affected & 94 & 89 \\
Frontal + parietal & 78 & 47 \\
Frontal + temporal & 67 & 53 \\
Parietal + temporal & 61 & 42 \\
Occipital + parietal & 61 & 32 \\
$\geq 3$ Regions affected & 89 & 68 \\
Frontal $+\geq 2$ neocortical & 42 \\
Occipital $+\geq 2$ neocortical & 67 & 26 \\
Frontal + parietal + temporal $^{*}$ & 61 & 32 \\
Occipital + parietal + frontal $^{*}$ & 56 & 26 \\
Hippocampus $+\geq 2$ neocortical $^{*}$ & 56 & 42 \\
\hline
\end{tabular}

Note:-DWI indicates diffusion-weighted imaging; FLAIR, fluid-attenuated inversion recovery.

* Two of frontal/temporal/parietal/occipital.

The codon 129 genotype distributions and EEG and CSF findings did not differ significantly between both groups.

In previous studies, basal ganglia abnormalities on T2WI were shown to correlate with rapidly progressive dementia and short disease duration, whereas in patients lacking this finding, the onset of dementia was delayed and the disease course was prolonged. ${ }^{22,23}$ Using highly sensitive sequences, we were now able to identify isolated cortex involvement on DWI as a main lesion pattern in CJD correlating with prolonged disease duration. The findings on DWI at the time point of the diagnosis may thus indicate a rather slowly or rapidly progressive disease course. Although the presence of basal ganglia hyperintensity, on the whole, may represent a negative prognostic factor, there may be single disease variants (determined by codon 129 genotype of the PRNP and the type of $\mathrm{PrP}^{\mathrm{Sc}}$ with prolonged disease duration showing basal ganglia lesions on MR imaging. ${ }^{14}$

Because patients with CJD with only cortical hyperintensities and atypical clinical presentation may be diagnosed with delay, the risk of disease transmission through invasive diagnostic procedures, such as brain biopsies, is present. CJD should, therefore, be considered as well in cases of slowly progressive dementia without characteristic basal ganglia abnormalities. Follow-up MR imaging examinations should be performed because they may show characteristic basal ganglia lesions in the later disease stage. ${ }^{24}$

Isolated cortex involvement on DWI has been reported in other conditions, such as encephalitis, ${ }^{25-27}$ hypoxia, ${ }^{28}$ and epilepsy. ${ }^{29-32}$ In these cases, further radiologic signs, such as cortical swelling, contrast enhancement on T1-weighted images, absence of hyperintensities on conventional images, predilection sites (hippocampus), and the partly observed resolution of the findings during the disease course may be useful discriminating factors from CJD.

In other cognitive disorders, used in the main differential diagnosis of CJD, isolated involvement of the neocortex was found in $17 \%$ of patients, mainly in the limbic cortex $(25 \%)$ but also in the temporal (11\%) and frontal lobes $(9 \%)$. The parietal and occipital lobes were rarely affected (3\% and 2\%) (lesions not assigned to a single diagnosis). ${ }^{9}$ As a major distinctive criterion, patients with CJD in our study showed more widespread cortex involvement on DWI, which typically included the frontal and parietal lobes (78\%). Similar cortical lesions, particularly on DWI, have, to our knowledge, not been described in other neurodegenerative diseases and may thus be useful in differentiating CJD from other dementias.

No MR imaging abnormalities apart from atrophy were detected in 4 of our patients with variable disease duration. Single reported cases with negative findings on DWI imply that the absence of signal-intensity alterations may be related to a very early or late examination time point ${ }^{33,34}$ or the findings may be too subtle or hard to differentiate from artifacts. ${ }^{9,35}$ Negative findings on all applied MR images have been reported in a rare variant of sporadic CJD. ${ }^{16,17}$ The CSF was positive for the 14-3-3 protein in 3 of our patients with normal MR imaging findings and may thus be a useful complementary diagnostic investigation.

In 8 patients in our study showing abnormalities only on DWI, the frontal, temporal, and parietal lobes were affected. Because DWI has already been reported to show signal-intensity alterations early in the disease course, ${ }^{33}$ this finding might reflect the onset of the disease in these brain areas and might match the early neuropsychologic deficits observed in patients with CJD. ${ }^{36,37}$

DWI, in contrast to FLAIR, depicted a significantly higher rate of high signals in the frontal, temporal, parietal, and occipital lobes as well as in the insula. This discrepancy may be explained by the neuropathologic lesions underlying the signal-intensity changes: Only predominant gliosis with a lower degree of spongiform changes gave high signals on T2WI in a hamster model. ${ }^{6}$ Because FLAIR is actually a T2WI MR imaging (without free fluids giving signal intensity), it most likely shows gliotic changes, as well. In contrast, DWI abnormalities are most probably caused by spongiform degeneration leading to decreased water diffusion due to the small size of the vacuoles. ${ }^{4,5}$ We assume that vacuoles are the predominant neuropathologic feature in the early disease stage, correlating with higher DWI sensitivity. Reactive gliotic changes may become more prominent during the disease course, causing high signals on FLAIR. Serial MR imaging studies with lesions detected first by DWI and only later by FLAIR and T2WI support this hypothesis. ${ }^{24,38,39}$

A correlation between widespread cortical hyperintensity on FLAIR and prolonged disease duration was found in the patients in our study. This finding supports the idea that widespread gliotic changes causing high signals on FLAIR are rather found in the advanced disease stages. Concerning DWI, no such correlation was found, which may be due to the small patient number with only little cortex involvement on DWI in our study. 
An influence of the codon 129 genotype of the PRNP and the $\mathrm{PrP}^{\mathrm{Sc}}$ isotype 1 or 2 (molecular subtype) on MR imaging findings in CJD has been postulated in small case series. ${ }^{13-17}$ Because, as in our study, the overall genotype distribution did not differ significantly between patients with and without basal ganglia signal-intensity increase on MR imaging, we assume that the $\mathrm{PrP}^{\mathrm{Sc}}$ isotype may represent a major influencing factor on the neuropathologic lesion distribution and, consecutively, the signal intensity alteration patterns on the MR imaging. The role of the molecular subtype concerning MR imaging lesion patterns in CJD should be evaluated in further studies including sufficient case numbers for each type.

\section{Conclusions}

The proportion of patients with CJD presenting without basal ganglia abnormalities is high, even when DWI is applied. Isolated cortical hyperintensities, as seen in one third of the patients, should be recognized as a frequently occurring pattern on the MR imaging, which might be characteristic of atypical disease variants with a slowly progressive course. Involvement of the frontal and parietal lobes as a consistent finding on FLAIR and DWI may support the CJD diagnosis. CSF analysis for the 14-3-3 protein may represent a useful complementary test in patients without signal-intensity abnormalities or with isolated cortical hyperintensity on MR imaging.

\section{Acknowledgments}

We thank all the physicians who reported suggestive cases to the German CJD Surveillance Unit for providing pertinent clinical and especially neuroradiologic data. We also thank Dr. Walter Schulz-Schaeffer and Prof. Hans Kretzschmar for contributing neuropathologic data and Jolanthe Ehrlich, Maja Schneider-Dominco, and Christine Crozier for the valuable assistance in the data management and editing of the manuscript.

\section{References}

1. Zerr I, Schulz-Schaeffer WJ, Giese A, et al. Current clinical diagnosis in CJD: identification of uncommon variants. Ann Neurol 2000;48:323-29

2. Parchi P, Castellani R, Yuan J, et al. Molecular basis of phenotypic variability in sporadic Creutzfeldt-Jakob disease. Ann Neurol 1996;39:767-78

3. Parchi P, Giese A, Capellari S, et al. Classification of sporadic CreutzfeldtJakob disease based on molecular and phenotypic analysis of 300 subjects. Ann Neurol 1999;46:224-33

4. Mittal S, Farmer P, Kalina P, et al. Correlation of diffusion-weighted magnetic resonance imaging with neuropathology in Creutzfeldt-Jakob disease. Arch Neurol 2002;59:128-34

5. Na DL, Suh CK, Choi SH, et al. Diffusion-weighted magnetic resonance imaging in probable Creutzfeldt-Jakob disease: a clinical-anatomic correlation. Arch Neurol 1999;56:951-57

6. Chung Y-L, Williams A, Ritchie D, et al. Conflicting MRI signals from gliosis and neuronal vacuolation in prion diseases. Neuroreport 1999;10:3471-77

7. Shiga Y, Miyazawa K, Sato S, et al. Diffusion-weighted MRI abnormalities as an early diagnostic marker for Creutzfeldt-Jakob disease. Neurology 2004;63: 443-49

8. Tschampa HJ, Kallenberg K, Urbach $\mathrm{H}$, et al. MRI in the diagnosis of sporadic Creutzfeldt-Jakob disease: a study on inter-observer agreement. Brain 2005;128:2026-33

9. Young GS, Geschwind MD, Fischbein NJ, et al. Diffusion-weighted and fluidattenuated inversion recovery imaging in Creutzfeldt-Jakob disease: high sensitivity and specificity for diagnosis. AJNR Am J Neuroradiol 2005;26:1551-62

10. Kallenberg K, Schulz-Schaeffer W, Jastrow U, et al. Creutzfeldt-Jakob disease:
Comparative analysis of MR imaging sequences. AJNR Am J Neuroradiol 2006;27:1459-62

11. Tschampa HJ, Kallenberg K, Kretzschmar HA, et al. Pattern of cortical changes in sporadic Creutzfeldt-Jakob disease. AJNR Am J Neuroradiol 2007;28: 1114-18

12. Collins SJ, Sanchez-Juan P, Masters CL, et al. Determinants of diagnostic investigation sensitivities across the clinical spectrum of sporadic CreutzfeldtJakob disease. Brain 2006;129:2278-87

13. Meissner B, Westner IM, Kallenberg K, et al. Sporadic Creutzfeldt-Jakob disease: clinical and diagnostic characteristics of the rare VV1 type. Neurology 2005; $65: 1544-50$

14. Krasnianski A, Schulz-Schaeffer WJ, Kallenberg K, et al. Clinical findings and diagnostic tests in the MV2 subtype of sporadic CJD. Brain 2006;129:2288-96

15. Fukushima R, Shiga Y, Nakamura M, et al. MRI characteristics of sporadic CJD with valine homozygosity at codon 129 of the prion protein gene and PrPSc type 2 in Japan. J Neurol Neurosurg Psychiatry 2004;75:485-87

16. Krasnianski A, Meissner B, Schulz-Schaeffer WJ, et al. Clinical features and diagnosis of the MM2 cortical subtype of sporadic Creutzfeldt-Jakob disease. Arch Neurol 2006;63:876-80

17. Hamaguchi T, Kitamoto T, Sato T, et al. Clinical diagnosis of MM2-type sporadic Creutzfeldt-Jakob disease. Neurology 2005;64:643-48

18. Human transmissible spongiform encephalopathies. Wkly Epidemiol Rec 1998;47:361-65

19. Windl O, Giese A, Schulz-Schaeffer W, et al. Molecular genetics of human prion diseases in Germany. Hum Genet 1999;105:244-52

20. Steinhoff BJ, Räcker S, Herrendorf G, et al. Accuracy and reliability of periodic sharp wave complexes in Creutzfeldt-Jakob disease. Arch Neurol 1996;53:162-66

21. Zerr I, Pocchiari M, Collins S, et al. Analysis of EEG and CSF 14-3-3 proteins as aids to the diagnosis of Creutzfeldt-Jakob disease. Neurology 2000;55:811-15

22. Meissner B, Köhler K, Körtner K, et al. Sporadic Creutzfeldt-Jakob disease magnetic resonance imaging and clinical findings. Neurology 2004;63:450-56

23. Urbach H, Klisch J, Wolf HK, et al. MRI in sporadic Creutzfeldt-Jakob disease correlation with clinical and neuropathological data. Neuroradiology 1998;40:65-70

24. Ukisu R, Kushihashi T, Kitanosono T, et al. Serial diffusion-weighted MRI of Creutzfeldt-Jakob disease. AJR Am J Roentgenol 2005;184:560-66

25. Gorniak RJ, Young GS, Wiese DE, et al. MR imaging of human herpesvirus-6associated encephalitis in 4 patients with anterograde amnesia after allogeneic hematopoietic stem-cell transplantation. AJNR Am J Neuroradiol 2006;27:887-91

26. Yoshinari S, Hamano S, Ito T, et al. MRI-diffusion-weighted images of encephalopathy associated with human herpesvirus 6 infection [in Japanese]. No To Hattatsu 2005;37:374-79

27. Küker W, Nägele T, Schmidt F, et al. Diffusion-weighted MRI in herpes simplex encephalitis: a report of three cases. Neuroradiology 2004;46:122-25

28. Arbelaez A, Castillo M, Mukherji SK. Diffusion-weighted MR imaging of global cerebral anoxia. AJNR Am J Neuroradiol 1999;20:999-1007

29. Kawada J, Kimura H, Yoshikawa T, et al. Hemiconvulsion-hemiplegia syndrome and primary human herpesvirus 7 infection. Brain Dev 2004;26:412-14

30. Calistri V, Caramia F, Bianco F, et al. Visualization of evolving status epilepticus with perfusion and diffusion MR imaging. AJNR Am J Neuroradiol 2003;24:671-73

31. Kim JA, Chung JI, Yoon PH, et al. Transient MR signal changes in patients with generalized tonicoclonic seizure or status epilepticus: periictal diffusionweighted imaging. AJNR Am J Neuroradiol 2001;22:1149-60

32. Chan S, Chin SS, Kartha K, et al. Reversible signal abnormalities in the hippocampus and neocortex after prolonged seizures. AJNR Am J Neuroradiol 1996;17:1725-31

33. Tomita I, Sato K, Shirabe S, et al. Serial diffusion-weighted MRI (DWI) in a patient with sporadic Creutzfeldt-Jakob disease [in Japanese]. Rinsho Shinkeigaku 2004;44:182-86

34. Tribl GG, Strasser G, Zeitlhofer J, et al. Sequential MRI in a case of CreutzfeldtJakob disease. Neuroradiology 2002;44:223-26

35. Alvarez FJ, Bisbe J, Bisbe V, et al. Magnetic resonance imaging findings in preclinical Creutzfeldt-Jakob disease. Int J Neurosci 2005;115:1219-25

36. Cordery RJ, Alner K, Cipolotti L, et al. The neuropsychology of variant CJD: a comparative study with inherited and sporadic forms of prion disease. J Neurol Neurosurg Psychiatry 2005;76:330-36

37. Pachalska M, Kurzbauer H, MacQueen BD, et al. Neuropsychological features of rapidly progressive dementia in a patient with an atypical presentation of Creutzfeldt-Jakob disease. Med Sci Monit 2001;7:1307-15

38. Matoba M, Tonami $\mathrm{H}$, Miyaji $\mathrm{H}$, et al. Creutzfeldt-Jakob disease: serial changes on diffusion-weighted MRI. J Comput Assist Tomogr 2001;25:274-77

39. Kropp S, Finkenstaedt M, Zerr I, et al. Diffusion-weighted MRI in patients with Creutzfeldt-Jakob disease [in German]. Nervenarzt 2000;71:91-95 